Check for updates

Cite this: RSC Adv., 2017, 7, 45304

Received 22nd August 2017

Accepted 15th September 2017

DOI: $10.1039 / c 7 r a 09303 a$

rsc.li/rsc-advances

\section{Schottky defects induced effects on the behaviors of high velocity shock compression of $\mathrm{MgO}$}

\author{
Chuanguo Zhang, (D) ${ }^{\text {ad }}$ Xianlong Wang, ${ }^{\text {ab }}$ Jie Zhang, $^{\text {ab }}$ Kaishuai Yang, ${ }^{\text {ab }}$ Ya Cheng, ${ }^{\text {ab }}$ \\ Zhi Zeng, ${ }^{\text {*abd }}$ Xianming Zhou ${ }^{c}$ and Haiqing Lin ${ }^{d}$
}

Molecular dynamics (MD) simulation was performed on high velocity plane shock compression of sodium chloride $\mathrm{MgO}$ along [100] lattice direction using a long-range coulombic potential. It was found that the shock compressions of $\mathrm{MgO}$ revealed three distinct regions depending on piston velocities, i.e., single elastic shock wave, two-wave structure consisting of an elastic wave followed by a plastic deformation wave, and single plastic deformation shock wave. The critical pressure of $120 \mathrm{GPa}$ was obtained for the transition from elastic to plastic deformation under the high strain rate of $10^{10} \mathrm{~s}^{-1}$. For getting a better understanding the experimental results, we also investigated the effects of Schottky defects in $\mathrm{MgO}$ single crystal on the Hugoniot Elastic Limit (HEL), the velocity of shock wave and the critical piston velocity (CPV) resulting in plastic deformation. Our results demonstrated that the HEL decreases from $120 \mathrm{GPa}$ to $100 \mathrm{GPa}$, and the CPV decreases a little from $2.8 \mathrm{~km} \mathrm{~s}^{-1}$ to $2.2 \mathrm{~km} \mathrm{~s}^{-1}$. Significantly, a double yielding phenomenon occurs for shock wave propagating in $\mathrm{MgO}$ sample with Schottky defects, i.e., homogeneous nucleation of dislocations accompanied by vacancy emission of dislocations, which has never been reported before. This work helps to clarify the contradictory results of $\mathrm{HEL}$ of MgO in the literature and to understand the plastic deformation mechanism of $\mathrm{MgO}$.

\section{Introduction}

Magnesium oxide ( $\mathrm{MgO})$ is an abundant mineral in the Earth's lower mantle, ${ }^{1}$ therefore, its high-temperature and highpressure properties are critical for understanding the Earth's interiors sufficiently. Furthermore, $\mathrm{MgO}$ is also widely used as pressure scale in static compression experiments ${ }^{2-4}$ due to its high stability at extreme conditions, high-temperature and high-pressure. Thus, it is required to obtain an accurate description of the equation of state (EOS) of MgO. To discuss the EOS of MgO from the Hugoniot data, it is necessary to clarify the effect of shear strength of material which usually can be discussed based on its Hugoniot Elastic Limit (HEL) during shock. However, to date, the HEL of MgO along crystallographic [100] direction is still in contradiction in the literatures. ${ }^{5-8}$ The reason for the experimentally reported controvertial MgO HELs are still unknown. Both strain rate and defects of $\mathrm{MgO}$ may affect the measured HELs.

Plane shock loading on $\mathrm{MgO}$ experimentally showed the splitting of shock waves, ${ }^{7}$ i.e., an elastic wave followed by

${ }^{a}$ Key Laboratory of Materials Physics, Institute of Solid State Physics, Chinese Academy of Sciences, Hefei 230031, China. E-mail: zzeng@theory.issp.ac.cn; Fax: +86-055165591434; Tel: +86-0551-65591407

${ }^{b}$ University of Science and Technology of China, Hefei 230026, China

${ }^{c}$ Institute of Fluid Physics, National Key Laboratory of Shock Wave and Detonation Physics, Mianyang 621900, China

${ }^{d}$ Beijing Computational Science Research Center, Beijing 100084, China a plastic deformation wave. Within the pressure of 1.4 terapascals (TPa), two phase transitions were observed by shock compression experiments: ${ }^{9} \mathrm{MgO}$ firstly transfers from sodium chloride to cesium chloride crystal at $0.36 \mathrm{TPa}$ while a metallic liquid phase appears under the pressure higher than 0.60 TPa. Despite great importance in technology and nature of $\mathrm{MgO}$, many open questions remain, mostly related to the underlying atomistic processes, such as plastic deformation mechanism. It is a challenging subject to experimentally explore the microscopic information including plastic deformation mechanisms during shock loading process. Computer material simulations are particularly useful and powerful for problems that may be inaccessible to direct experimental studies, such as extreme of pressure. MD simulations and density functional theory (DFT) were widely used to clarify the $\mathrm{MgO}$ properties, such as melting mechanism, ${ }^{\mathbf{1 0 - 1 2}}$ equation of state of $\mathrm{MgO}^{\mathbf{1 3}}$ and phase diagram. ${ }^{14}$ Within our best knowledge, up to now, there is no MD simulation about the $\mathrm{MgO}$ shock compression due to its complicate behaviors. Moreover, despite that defects have significant effects on dynamic materials properties and processes, ${ }^{\mathbf{1 5 - 1 8}}$ including high strain rate loading, studies along this line on $\mathrm{MgO}$ are rare. On the one hand, high level concentration of vacancies will form in materials serviced under extreme environment due to the irradiation of neutrons or highenergy particles. ${ }^{19}$ On the other hand, the effects of defects in materials are normally entangled and it is desirable to separate their individual contributions in a research. In this work, using 
MD simulations, we illustrate the simplest individual case, the effects of Schottky defects on the shock response of MgO single crystal. Understanding the mechanical deformation of $\mathrm{MgO}$ with Schottky defects should help in the design of materials with improved mechanical properties.

There are several literatures focusing on a single void collapse ${ }^{16,17,20-24}$ or a collection of voids interaction ${ }^{25}$ in both face-centered cubic (fcc) or body-centered cubic (bcc) metals at high strain rates. Dislocations induced by loading are emitted from void surfaces as shear loops, with their interactions leading to hardening. ${ }^{24}$ The individual void serves as dislocation sources, whose efficiency is further enhanced by their collective interaction eventually leading to very high dislocation densities. $^{25}$ This work focuses on dislocation activity and the resulting stress relaxation in $\mathrm{MgO}$ single crystal sample with Schottky defects, which has not been discussed in depth. ${ }^{15}$ We have studied the effects of Schottky defects on the elastic-plastic transition of $\mathrm{MgO}$ single crystal under high strain rate shock compression by large scale MD simulations, in order to elucidate the influence of Schottky defects on the shock wave propagation and the deformation mechanism of MgO. Our results indicate that Schottky defects in $\mathrm{MgO}$ single crystal can affect the HEL, and a double yielding phenomenon occurs for shock wave propagating in $\mathrm{MgO}$ sample with Schottky defects. This work clarify the contradictory results of MgO HEL in the literature and present the plastic deformation mechanism of MgO.

\section{Methodology of the simulations}

The dynamics of shock wave propagation along [100] lattice direction in $\mathrm{MgO}$ single crystal at $1000 \mathrm{~K}$ are investigated with large-scale non-equilibrium MD simulations performed with software package LAMMPS. ${ }^{26}$ The simulated specimens are about $84.336 \mathrm{~nm}, 16.952 \mathrm{~nm}$ and $16.952 \mathrm{~nm}$ along the Cartesian $x$-, $y$-, and $z$-axes containing 2547200 atoms. A small slab model $(63.146 \mathrm{~nm} \times 16.952 \mathrm{~nm} \times 16.952 \mathrm{~nm}$, containing 1907200 atoms) is also created to eliminate the size effects. All simulations are performed with periodic boundary conditions in $x, y$ and $z$ directions, and a vacuum layer of $4.662 \mathrm{~nm}$ is created along shock direction. Plane shocks are simulated along $x$-axis with [100] lattice direction. The plane shock waves are produced by slamming a piston with a certain velocity, i.e., the piston velocity $\left(U_{\mathrm{p}}\right)$, and propagate with velocity $U_{\mathrm{s}}$ in $\mathrm{MgO}$ material. The piston velocity $U_{\mathrm{p}}$ is equal to the particle velocity $\left(u_{\mathrm{p}}\right)$ behind the shock wave. Velocity profiles are analyzed to obtain $U_{\mathrm{s}}$. The relationship between $U_{\mathrm{p}}$ and $U_{\mathrm{s}}$ is represented in the Hugoniot curve. The shock profiles which include particle velocity, stresses, mass density and atomic displacement in the $y z$ plane are calculated along the system in the $x$ direction.

Identification of defects is done using the centrosymmetry parameter ${ }^{27}$ and the dislocation extraction algorithm (DXA). ${ }^{28}$ The latter enables one to identify line and surface defects and to measure dislocation densities. Visualization and analysis of atomistic simulation data are performed using OVITO. ${ }^{29}$ The prerequisite condition for the success of MD simulation is the availability of reliable interatomic potentials describing the interaction between atoms in the crystalline lattice. The
Table 1 Short-range potential parameters of $\mathrm{MgO}$ used in simulations

\begin{tabular}{lrll}
\hline & \multicolumn{1}{c}{$A(\mathrm{eV})$} & $\rho(\mathrm{nm})$ & $C\left(\mathrm{eV} \mathrm{nm}^{6}\right)$ \\
\hline $\mathrm{Mg}-\mathrm{Mg}$ & 0.0 & 0.1000 & 0.0 \\
$\mathrm{Mg}-\mathrm{O}$ & 821.6 & 0.03242 & 0.0 \\
$\mathrm{O}-\mathrm{O}$ & 22764.4 & 0.01490 & $2.037 \times 10^{-5}$
\end{tabular}

empirical potential form ${ }^{30}$ is applied according to the work of Lewis and Catlow, as shown in the following expression:

$$
E_{i j}=\frac{Z_{i} Z_{j} e^{2}}{r_{i j}}+A_{i j} \exp \left(-\frac{r_{i j}}{\rho_{i j}}\right)-\frac{C_{i j}}{r_{i j}},
$$

where $E_{i j}$ is the interatomic potential, the right-hand side terms represent the Coulomb interaction, repulsion energy, and van der Waals force, respectively. Here $A_{i j}$ and $\rho_{i j}$ are the parameters for the repulsive interaction, $C_{i j}$ is van der Waals constant, $Z_{i}$ or $Z_{j}$ is an effective charge with $\mathrm{Mg}$ ion of 2 and $\mathrm{O}$ ion of $-2, e$ is the electronic unit charge, $r_{i j}$ is the interatomic distance between atoms $i$ and $j$. The parameters of the short-range potential are shown in Table 1. We use the particle-particle particle-mesh (PPPM) method, which is more efficient than the standard Ewald summation, as the $k$-space solver of the long-range coulombic interactions with a precision of $1.0 \times 10^{-5}$ and a cutoff distance of $0.8 \mathrm{~nm}$. The original potential as given in ref. 30 also includes a polarizable shell. However, in the MD simulations performed here we do not include shells, using instead of a rigid-ion approximation for simplicity. Despite of the simple form of the potential, the stress-strain curve of uniaxial compression of $\mathrm{MgO}$ along [100] lattice direction calculated via MD method is in good agreement with that of DFT method when the strain is less than 0.15 as shown in Fig. 1 . Furthermore the $P-V$ curve corresponds very well with the experimental results ${ }^{2,31,32}$ and the results of other computational models ${ }^{4,14}$ as shown in Fig. 2.

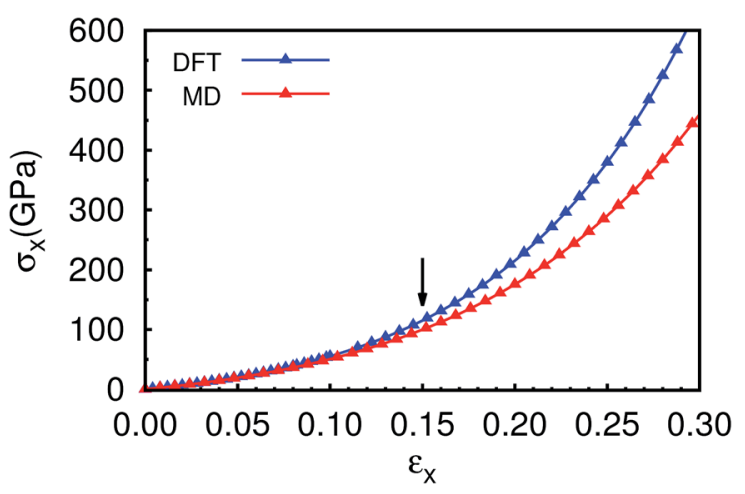

Fig. 1 The relationship between the normal pressure $\left(\sigma_{x}\right)$ and the strain $\left(\varepsilon_{x}\right)$ by uniaxial compression along [100] lattice direction compared to the result of DFT method. The black arrow indicates $\varepsilon_{x}$ with the value of 0.15 . The stress-strain curve calculated from MD method is in good agreement with that of DFT method when the strain is less than 0.15 . 

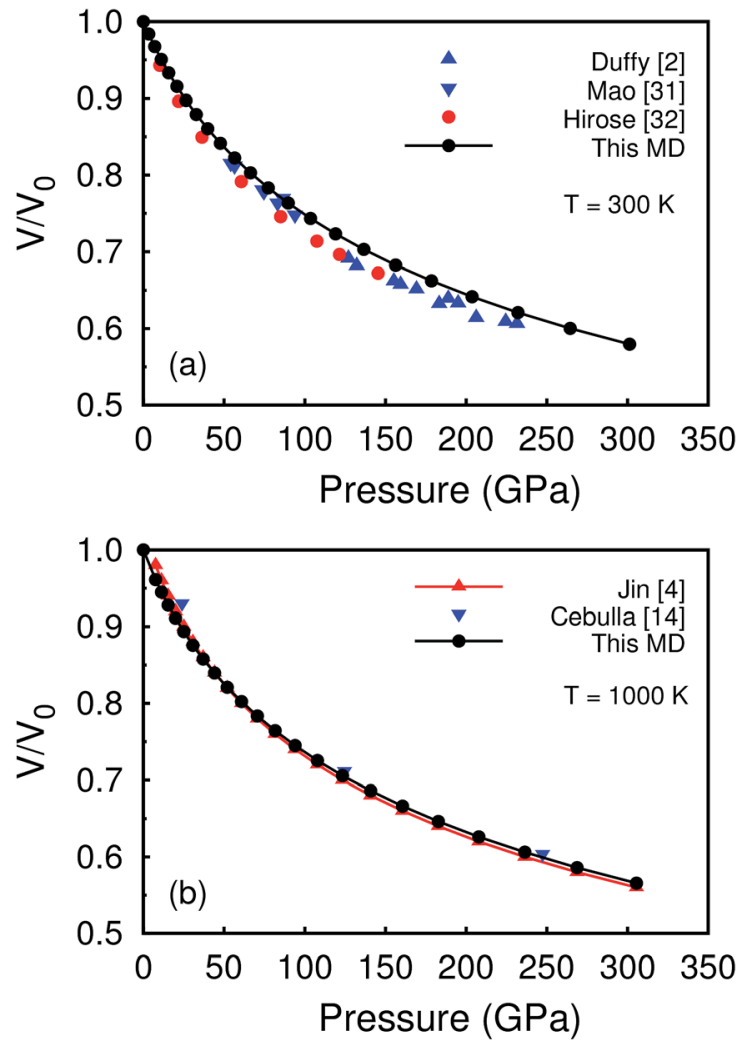

Fig. 2 The $P-V$ EOS of MgO calculated via MD method compared to the results of experiments and other computational models at (a) 300 $\mathrm{K}$ and (b) $1000 \mathrm{~K}$, respectively.

\section{Results and discussion}

\subsection{Hugoniot $U_{\mathrm{s}}-U_{\mathrm{p}}$ curve}

For particle velocity distribution as shown in Fig. 3(a), two wave structures are identified, i.e., an elastic wave is followed by a plastic wave, which has well reproduced the experimental detected phenomenon, ${ }^{7}$ where they also found two-wave structure along [100] crystallographic shock compression. The average displacement of atoms perpendicular to the impact direction is a useful quantity to distinguish elastic wave from plastic wave. In Fig. 3(b), the displacement in the $y z$ plane increases distinctly along the impact direction. The distribution of mass density also reflects the differences among undisturbed area, elastic area and the plastic deformation area. As shown in Fig. 3(b) blue points, the mass density is about $3.46 \mathrm{~g} \mathrm{~cm}^{-3}$ ahead the shock front, which is the mass density of $\mathrm{MgO}$ in ordinary state at $1000 \mathrm{~K}$, while it increases to $4.4 \mathrm{~g} \mathrm{~cm}^{-3}$ and $4.7 \mathrm{~g} \mathrm{~cm}^{-3}$ in the elastic area and plastic area, respectively. It is also noted from Fig. $3(\mathrm{c})$ that the maximum shear stress $(\tau)$ in the plastic deformation region releases to a value of $10 \mathrm{GPa}$ via dislocation nucleation and slipping of $\{110\}$ [110] system, and the profile of the longitude stress $\left(\sigma_{x}\right)$ also exhibits clearly three areas, i.e., the undisturbed area, the elastic area and the plastic area.

The shock front velocity $U_{\mathrm{s}}$ is defined by following the drop in the particle velocity profile as shown in Fig. 3(a). Fig. 4
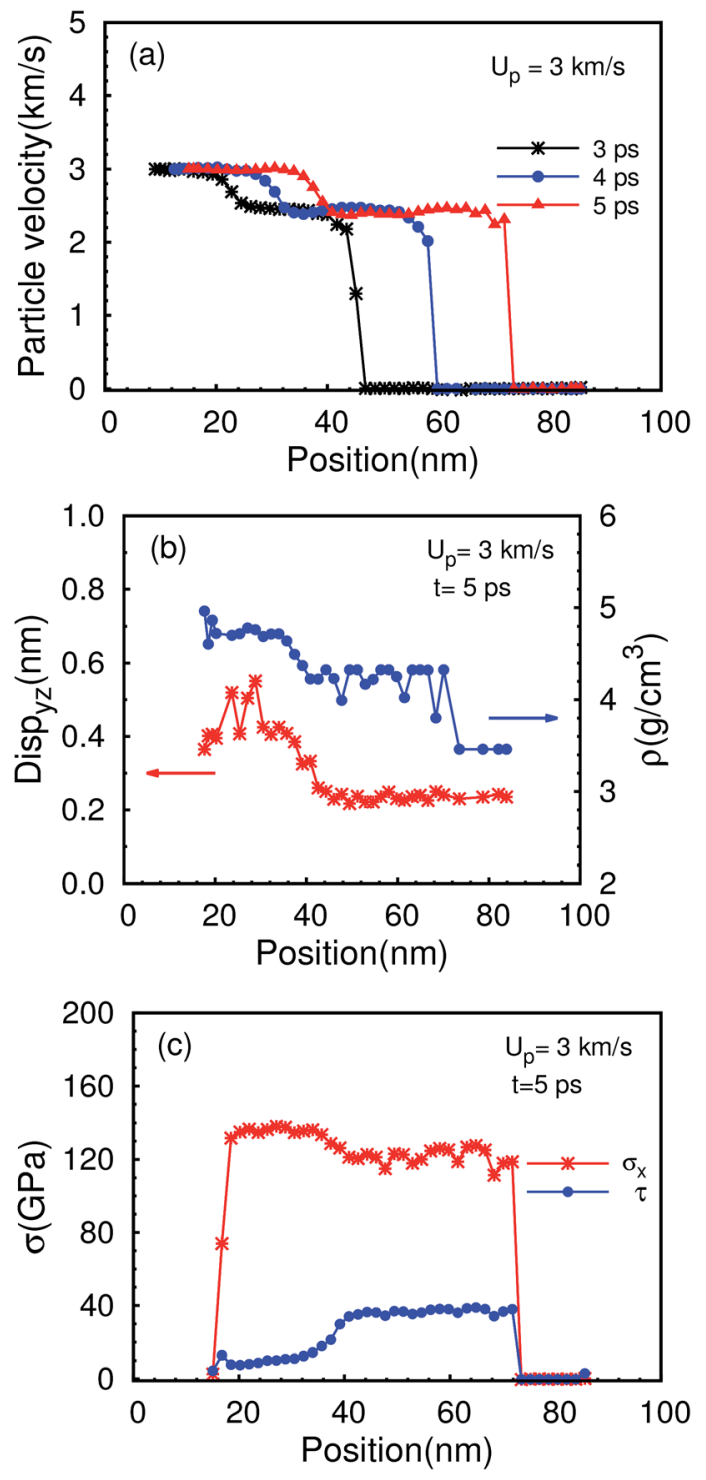

Fig. 3 (a) Profile of particle velocity. (b) Atomic displacement (the left vertical axis) in the $y z$ plane and mass density distribution (the right vertical axis) for $t=5 \mathrm{ps}$. Atomic displacement in the plastic deformation area is higher than that in the elastic area. (c) Profile of the longitude stress along shock direction $\left(\sigma_{x}\right)$ and the maximum shear stress $(\tau)$. The shock front moves from left to right with the piston velocity $U_{\mathrm{p}}=3.0 \mathrm{~km} \mathrm{~s}^{-1}$ along crystallographic [100] direction.

exhibits the Hugoniot $U_{\mathrm{s}}-U_{\mathrm{p}}$ relationship of the pristine MgO single crystal and the MgO single crystal with $0.1 \%$ Schottky defects along [100] crystallographic shock orientation. In this section, we only discuss the Hugoniot $U_{\mathrm{s}}-U_{\mathrm{p}}$ relationship of the pristine MgO single crystal, and leave the discussion on the Schottky defects to the next section. Note that $U_{\mathrm{p}}$ and $U_{\mathrm{s}}$ refer the piston velocity and the velocity of shock waves, respectively. We find that the shock compressions of MgO show three distinct regions depending on $U_{\mathrm{p}}$. At the beginning, when the piston owns a velocity below $2.8 \mathrm{~km} \mathrm{~s}^{-1}$, it only generates elastic deformation as indicated by the left red arrow in Fig. 4. Start from $2.8 \mathrm{~km} \mathrm{~s}^{-1}$ until $7.0 \mathrm{~km} \mathrm{~s}^{-1}$, a well-defined two-wave structure generates, consisting of an elastic wave followed by 


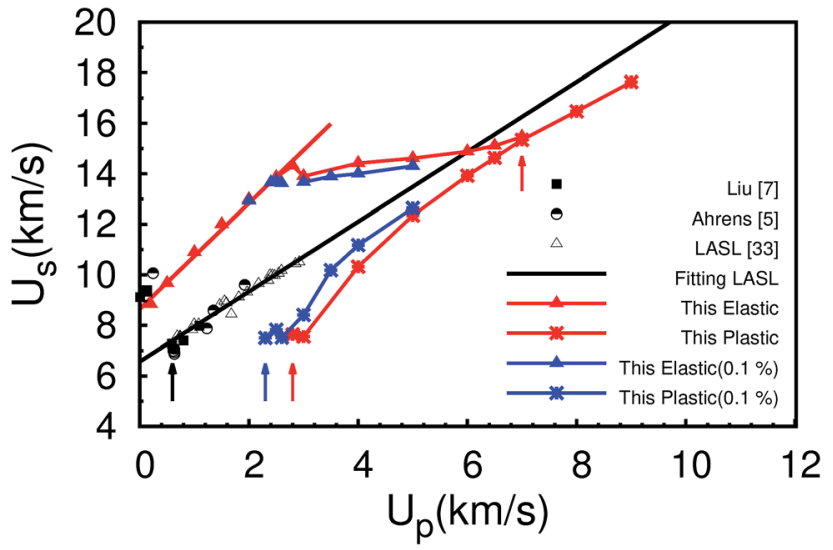

Fig. 4 The Hugoniot curve of MgO single crystal along [100] crystallographic shock orientation obtained by MD simulations at $1000 \mathrm{~K}$, compared to experimental data Liu, ${ }^{7}$ Ahrens ${ }^{5}$ and LASL, ${ }^{33}$ which were measured by the methods of velocity interferometer system for any reflector (VISAR), inclined-mirror and flash-gap, respectively. Red triangle points and red star points represent elastic shock wave and plastic deformation shock wave in the pristine $\mathrm{MgO}$ single crystal in our work; blue triangle points and blue star points represent elastic shock wave and plastic deformation shock wave in $\mathrm{MgO}$ single crystal with $0.1 \%$ Schottky defects. The right red arrow represents the piston velocity with which plastic wave velocity exceeds elastic wave velocity. The black arrow, the left red arrow and the blue arrow represent the critical piston velocity (CPV) resulting in plastic deformation for the experimental results ${ }^{5}$ and our MD simulations of the pristine $\mathrm{MgO}$ single crystal and the $\mathrm{MgO}$ single crystal with $0.1 \%$ Schottky defects, respectively.

a plastic deformation wave. Then when the piston velocity goes above $7.0 \mathrm{~km} \mathrm{~s}^{-1}$, as indicated by the right red arrow in Fig. 4, the plastic deformation shock wave overdrives the single elastic shock wave. Usually, the formula, $U_{\mathrm{s}}=C_{0}+\lambda u_{\mathrm{p}}$, describes the relationship between velocities of shock waves and particle velocities behind the shock front within limits. Here $C_{0}$ represents the velocity of elastic longitudinal wave, $u_{\mathrm{p}}$ represents the particle velocity which is the same as the piston velocity $\left(U_{\mathrm{p}}\right), \lambda$ is a constant. Consequently, we get the velocity of longitudinal elastic wave $C_{0}\left(8.65 \mathrm{~km} \mathrm{~s}^{-1}\right)$ and the constant $\lambda$ (2.094) by fitting the data of $U_{\mathrm{s}}$ and $U_{\mathrm{p}}$ in the region of single elastic shock wave following this linear function. We should be aware of that $C_{0}$ is consistent with sound velocity $\left(9.12 \mathrm{~km} \mathrm{~s}^{-1}\right)$ calculated from the elastic constant of $\mathrm{MgO} .{ }^{34}$ For the region of two-wave structure, it is interesting that the velocity of elastic wave increases slightly with increase of the piston velocity. Two obvious characteristics are obtained from Fig. 4 by comparing the Hugoniot $U_{\mathrm{s}}-U_{\mathrm{p}}$ relationship of the pristine $\mathrm{MgO}$ single crystal and the experimental results. First, the velocity of the plastic wave is much lower than that of the experimental results for the case of lower piston velocities in particular, whereas the difference will gradually decrease to about $1.0 \mathrm{~km} \mathrm{~s}^{-1}$ with increase of piston velocity. Second, the pristine $\mathrm{MgO}$ sample has a much higher value of the CPV compared to that of the experimental results, i.e., $2.8 \mathrm{~km} \mathrm{~s}^{-1}$ indicated by the left red arrow relative to $0.6-0.8$ $\mathrm{km} \mathrm{s}^{-1}$ indicated by the back arrow. Possible reasons for these differences are the strain rate of compression and the effects of defects in $\mathrm{MgO}$ materials.

\subsection{Hugoniot elastic limit of MgO under [100] shock compression}

For clarifying the critical pressure of elastic and plastic deformation, the shock Hugoniot $P-V$ curve is presented in Fig. 5. We can find that an inflection point exists at the $V / V_{0}=0.75$ corresponding to normal pressure of $120 \mathrm{GPa}$ and the piston velocity of $2.8 \mathrm{~km} \mathrm{~s}^{-1}$. The maximum shear stress $(\tau)$ decreases to near zero at the inflection point due to the plastic deformation, which indicates that the HEL of MgO is $120 \mathrm{GPa}$. While Liu et al. reported that the $\mathrm{HEL}$ of $\mathrm{MgO}$ under [100] shock direction were 4.3, 4.1, and $2.9 \mathrm{GPa}$ at final stresses of 16.3, 22.0, and 31.7 $\mathrm{GPa}$, respectively. ${ }^{7}$ It is acceptable that the $\mathrm{HEL}$ of $\mathrm{MgO}$ is as high as $120 \mathrm{GPa}$ under specific conditions. Sarva et al. used a modified split Hopkinson bar to perform the high strain rate tests in silicon carbide (SiC). ${ }^{35}$ Their results showed that the strain rate sensitivity of the compressive strength of SiC material was very strong. A marked increase is observed in the compressive strength at strain rates greater than $100 \mathrm{~s}^{-1}$. We evaluated the strain rate of our MD compression simulations, which is up to $10^{10} \mathrm{~s}^{-1}$, whereas the strain rate is only $10^{6} \mathrm{~s}^{-1}$ from the shock compression results of experimental group using bore-keyed powder gun method. Hence, it may be acceptable that the HEL is as high as $120 \mathrm{GPa}$ under the high strain rate of $10^{10} \mathrm{~s}^{-1}$. We note that a difference exists in the HEL of MgO measured by different experimental groups. ${ }^{5-8}$ This difference is likely due to the strain rate on the $\mathrm{MgO}$ samples during compression.

\subsection{The Hugoniot $U_{\mathrm{s}}-U_{\mathrm{p}}$ relationship and the HEL changing due to Schottky defects}

As mentioned above, one of the reasons to bring the discrepancy between experimental and simulated results might be from defects induced effects. We then focus on the effects of Schottky defects on the Hugoniot $U_{\mathrm{s}}-U_{\mathrm{p}}$ relationship and the HEL of MgO. When considering the effects of preexisting voids on dislocation nucleation in shocked fcc solids, Hatano found that the HEL drastically decreases to $15 \%$ of the perfect crystal

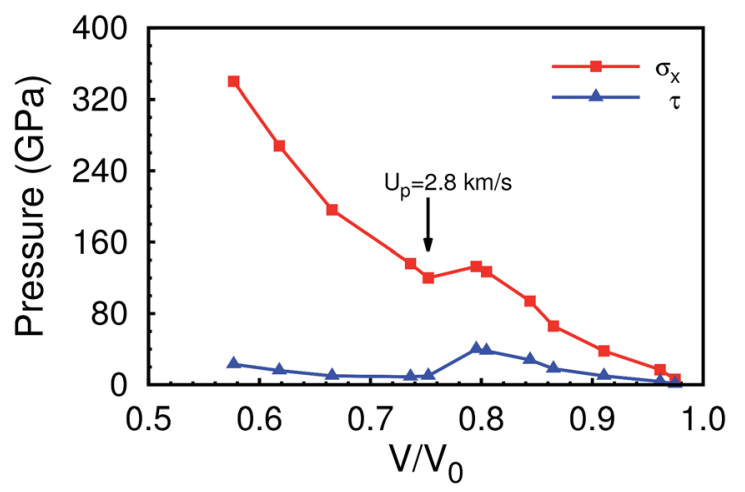

Fig. 5 The relationship between stress and volume. The maximum shear stress $(\tau)$ and normal pressure $\left(\sigma_{x}\right)$ along shock direction of [100] lattice direction. The black arrow indicates an inflection point at the $\mathrm{V} /$ $V_{0}=0.75$ corresponding to normal pressure of $120 \mathrm{GPa}$ and the piston velocity of $2.8 \mathrm{~km} \mathrm{~s}^{-1}$. 
when the void radius is $3.4 \mathrm{~nm} .^{36}$ One question is arising that how about the effects of Schottky defects in $\mathrm{MgO}$ on the Hugoniot $U_{\mathrm{s}}-U_{\mathrm{p}}$ relationship and the HEL. Normally the "equilibrium" Schottky defects concentration in $\mathrm{MgO}$ single crystal is very low at $1000 \mathrm{~K}$ and its effect is expected to be negligible. However, high vacancy concentration up to $1 \%$ can be metastably preserved via proper processing, e.g., thin film growth ${ }^{37}$ and ion irradiation. ${ }^{38}$ Furthermore, by studying the effects of Schottky defects, we can deduce which effects, the strain rate or the Schottky defects, play a dominant role in
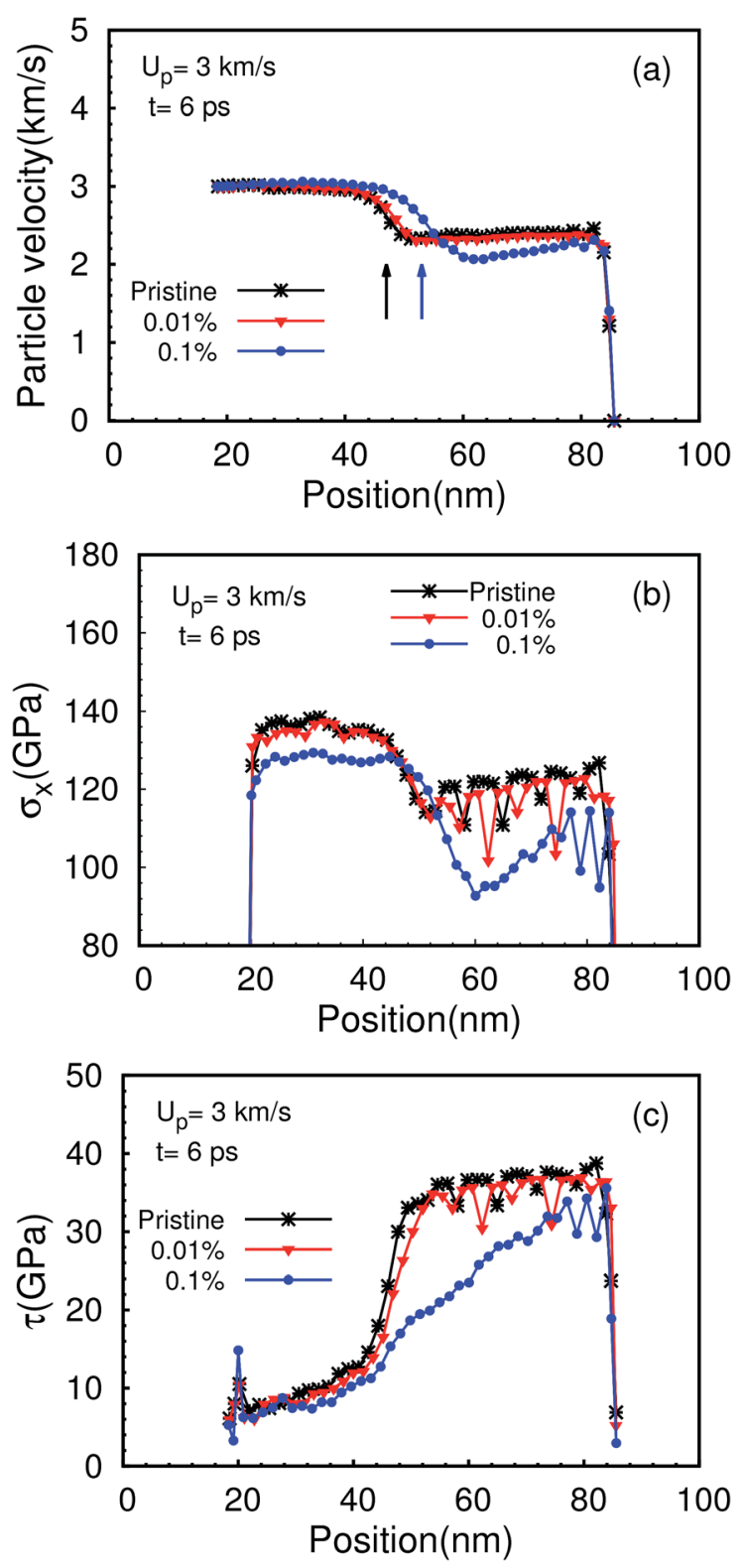

Fig. 6 The distributions of the particle velocity $\left(u_{p}\right)(a)$, the normal pressure $\left(\sigma_{x}\right)(\mathrm{b})$ and the maximum shear stress $(\tau)$ (c) with $U_{p}=3 \mathrm{~km}$ $\mathrm{s}^{-1}$ along [100] shock direction at $6 \mathrm{ps}$. The black points, red points and blue points represent the results of $\mathrm{MgO}$ single crystal with no Schottky defects (Pristine), $0.01 \%$ and $0.1 \%$ Schottky defects, respectively. The black arrow and the blue arrow indicate the positions of plastic deformation shock front of pristine $\mathrm{MgO}$ single crystal and $\mathrm{MgO}$ sample with $0.1 \%$ Schottky defects, respectively. changing of the $U_{\mathrm{s}}-U_{\mathrm{p}}$ relationship and the HEL of MgO. High vacancy concentration may facilitate considerably heterogeneous nucleation of plasticity and voids spall damage even within MD time and size scales. Hence we introduce Schottky defects of only $0.01 \%$ and $0.1 \%$ atomic concentration in the $\mathrm{MgO}$ single crystal to investigate the effects of defects. Fig. 6 shows the distribution of the particle velocity, the normal stress and the maximum shear stress of $\mathrm{MgO}$ single crystal without and with Schottky defects. Comparing the $\mathrm{MgO}$ impaction simulations, it presents the following characteristics. First, the velocity of plastic wave increases as indicated by the positions of those shock wave front in Fig. 6(a). Second, the stress strength of the shock wave decreases compared to that of the pristine $\mathrm{MgO}$ single crystal in Fig. 6(b). According to Minshall, ${ }^{39}$ the "normal pressure" of the elastic precursor is defined as the HEL. Therefore, our results demonstrated that the HEL decreases to $100 \mathrm{GPa}$ for the MgO sample with $0.1 \%$ Schottky defects. Third, the release of the maximum shear stress $(\tau)$ takes place in advance in Fig. 6 , which implies a new mechanism of plastic deformation for $\mathrm{MgO}$ sample with Schottky defects. We will discuss this new mechanism deeply in the next section. For comparison, we also plot the Hugoniot $U_{\mathrm{s}}-U_{\mathrm{p}}$ relationship of the $\mathrm{MgO}$ sample with $0.1 \%$ Schottky defects as shown in Fig. 4 . We can see that the velocity of elastic shock wave in MgO single crystal with $0.1 \%$ Schottky defects changes little compared to those of $\mathrm{MgO}$ single crystal without Schottky defects. The velocity of plastic wave increases and moves closer to the experimental Hugoniot data, but it still differs greatly with the experimental results reported by Ahrens ${ }^{5}$ and Marsh. ${ }^{33}$ We also note that the CPV resulting in plastic deformation decreases from $2.8 \mathrm{~km} \mathrm{~s}^{-1}$ (indicated by the left red arrow) to $2.2 \mathrm{~km} \mathrm{~s}^{-1}$ (indicated by the blue arrow). Furthermore, the HEL decreases from $120 \mathrm{GPa}$ to $100 \mathrm{GPa}$. Whereas the CPV and the HEL are still much higher than the experimental value of $0.6-0.8 \mathrm{~km} \mathrm{~s}^{-1}$ (ref. 5 and 7) and no more than $10 \mathrm{GPa}^{5-7}$ respectively. Therefore, the high value of the CPV and the HEL mainly result from the high strain rate of the shock compression compared to the effects of Schottky defects. Furthermore, even well annealed $\mathrm{Cu}$ single crystals have a dislocation density of $10^{5}$ to $10^{8} \mathrm{~cm}^{-2}$, leading to a low experimental HEL. ${ }^{40}$ Therefore, dislocation sources, such as Schottky defects, could be an alternative explanation to the departure of the calculated single crystal Hugoniot with respect to the experimental Hugoniot.

\subsection{Deformation mechanism of MgO with Schottky defects}

To understand the effects of Schottky defects on the Hugoniot $U_{\mathrm{s}}-U_{\mathrm{p}}$ relationship and the $\mathrm{HEL}$ of $\mathrm{MgO}$, we will discuss the deep insight of Schottky defects into the deformation mechanism of MgO. The decrease of shear stress corresponds to the plastic behavior, which is very complicate for $\mathrm{MgO}$ including dislocation activity, phase transformation and melting. Here we only focus on the plastic deformation mechanism of dislocation activity. The maximum shear stress $(\tau)$ decreases sharply as indicated by the black arrows in Fig. 7(a), while dislocations nucleate homogeneously when the shock wave travels through the material. Dislocations nucleate, grow and crisscross into a dislocation network, most of which are composed of $1 / 2\langle 110\rangle$ 

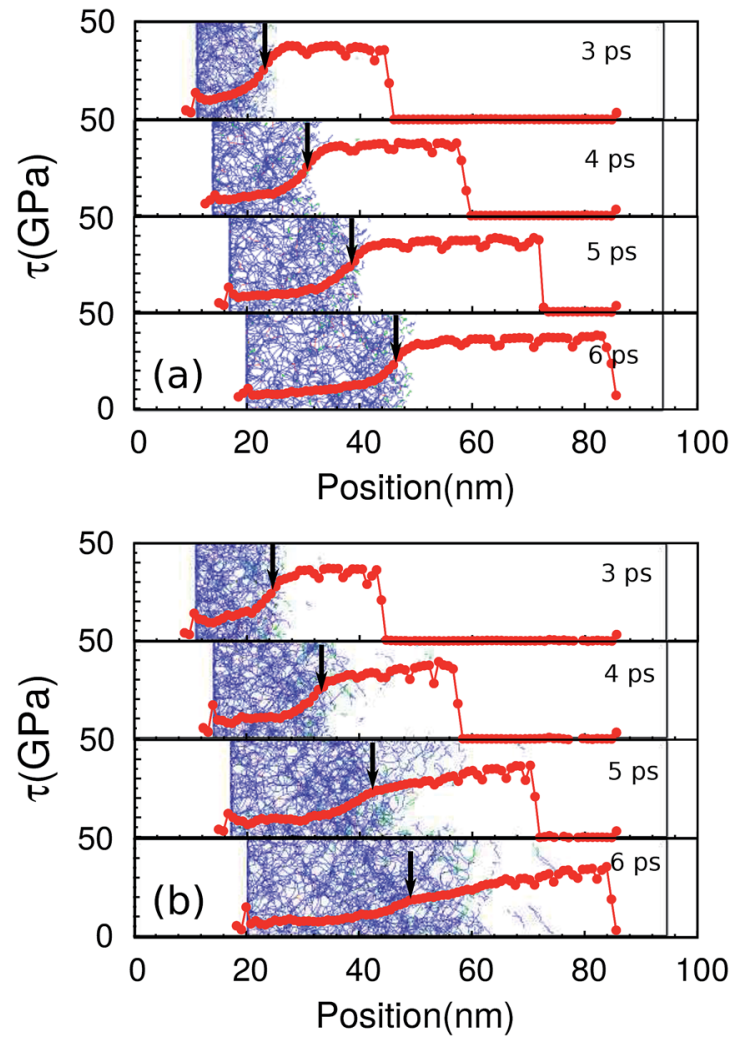

Fig. 7 The evolution of dislocation structures (blue lines) and the maximum shear stresses $(\tau)$ (red point line ranging from 0 to $50 \mathrm{GPa}$ ) in pristine $\mathrm{MgO}$ sample (a) and $\mathrm{MgO}$ single crystal with $0.1 \%$ (b). The shock front moves from left to right with the piston velocity of $3.0 \mathrm{~km} \mathrm{~s}^{-1}$. The black arrows indicate the positions of plastic deformation shock front

pristine dislocations, a small number of $1 / 6\langle 112\rangle$ Schottky dislocations as well as others. We also note that dislocationdislocation interactions leads to the development of a threedimensional pattern of intersecting loops in all available $\{110\}\langle 110\rangle$ slip planes with a large number of junctions. Most importantly, a double yielding phenomenon occurs in $\mathrm{MgO}$ sample with $0.1 \%$ Schottky defects, which is obviously different from the deformation behavior of the pristine $\mathrm{MgO}$ single crystal. Seeing the right part of the black arrow from Fig. 7(b), dislocations emitted from preexisting vacancies appear ahead of the shock front, which is in controversial to the clear region of Fig. 7(a). After initial stages of heterogeneous nucleation from the sources at $4-5 \mathrm{ps}$, there are two regions, that is the homogeneous and heterogeneous dislocation nucleation regions, divided by the black arrows in Fig. 7(b). The region of heterogeneous dislocation nucleations shows lower dislocation density than the region of homogeneous nucleation at $6 \mathrm{ps}$. Seen from the last frame of Fig. 7(b), the first slow decrease of the maximum shear stress corresponds to the vacancy emission dislocation, which has never been reported before, and the second decrease corresponds to the homogeneous nucleation of dislocations behind the shock front. The threshold stress of vacancy emission dislocation is less than that of dislocation homogeneous nucleation, leading to the vacancy emission of dislocations occurs first. Note that the width of shock front

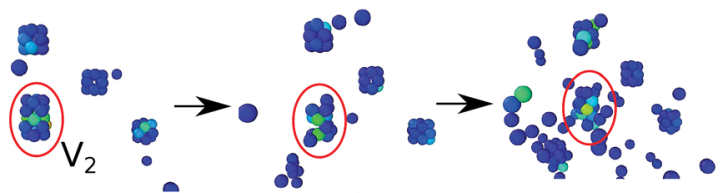

(a) $4.75 \mathrm{ps}$

(b) $5.0 \mathrm{ps}$

(c) $5.25 \mathrm{ps}$

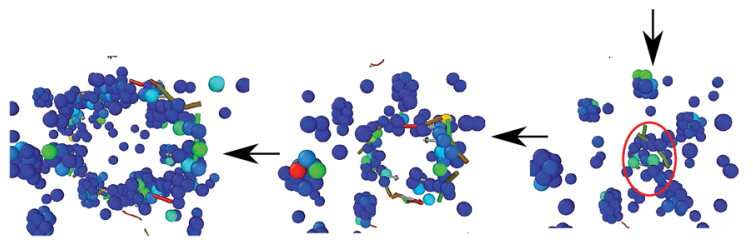

(f) $6.0 \mathrm{ps}$

(e) $5.75 \mathrm{ps}$

(d) $5.5 \mathrm{ps}$

Fig. 8 Series of snapshots of the $\mathrm{MgO}$ sample in the region of heterogeneous dislocation nucleations. The shock proceeds from the left to the right with $U_{p}=3.0 \mathrm{~km} \mathrm{~s}^{-1}$ in the $\mathrm{MgO}$ sample of $0.1 \%$ Schottky defects. The arrows show the evolution of atomistic configurations. The centrosymmetry parameter ${ }^{27}$ is used for color coding. Only defective $\mathrm{Mg}$ atoms (vacancy surfaces and dislocations) of a part of the $\mathrm{MgO}$ sample are shown with centrosymmetry parameter ranging from $0.05 \mathrm{~nm}^{2}$ to $0.28 \mathrm{~nm}^{2}$. Nucleation of dislocation from the surface of the two vacancies in the red circle $(a-c)$ is marked in the frame $(d)$ at $5.5 \mathrm{ps}$. The last three frames indicate the evolution of a dislocation loop, including the nucleation (d), growth (e) and expansion (f).

becomes wider compared to that of the pristine $\mathrm{MgO}$ single crystal sample due to this double yielding phenomenon. We find that vacancies (including monovacancies or bivacancies) serve as the nucleation sites for the shear planes and facilitate their growth. The elevated free energy and "free volume" associated with vacancies allow lower energy barrier for nucleation of local shear and the formation of dislocations. Fig. 8 illustrates the processes of dislocation nucleation, growth and expansion from a case of vacancy cluster $V_{2}$. First, positions of the atoms around $V_{2}$ are disturbed due to the shock compression from the frame (a) to (c). Then, a dislocation nucleates from the surface of the disturbed $V_{2}$ in the frame (d), grows gradually and finally forms a dislocation loop from the frame (e) to (f). As a result, the shear planes are irregularly spaced due to the randomness of vacancies. For the case of shock wave propagating in $\mathrm{MgO}$ sample with $0.01 \%$ Schottky defects, this double yielding phenomenon is not obvious. Therefore, a critical concentration of Schottky defects is required for localized shearing to emit dislocations from Schottky defects.

\section{Conclusion}

In summary, high velocity and strain rate shock compressions of $\mathrm{MgO}$ for [100] shock direction are investigated by MD simulations. We found that the shock compression of $\mathrm{MgO}$ shows three distinct regions, i.e., single elastic shock wave, two-wave structure consisting of an elastic wave followed by a plastic deformation wave and single plastic deformation shock wave. The results exhibit that the HEL of pristine MgO single crystal is $120 \mathrm{GPa}$ for crystallographic [100] direction under the high strain rate of $10^{10} \mathrm{~s}^{-1}$. We also investigated the effects of Schottky defects on the Hugoniot $U_{\mathrm{s}}-U_{\mathrm{p}}$ relationship and the 
HEL of MgO. First, the velocity of the plastic wave increases and moves closer to the experimental results. Second, the CPV decreases a little from $2.8 \mathrm{~km} \mathrm{~s}^{-1}$ to $2.2 \mathrm{~km} \mathrm{~s}^{-1}$. Third, the HEL decreases from $120 \mathrm{GPa}$ to $100 \mathrm{GPa}$. Whereas the CPV and the HEL are still much higher than the experimental value of 0.6$0.8 \mathrm{~km} \mathrm{~s}^{-1}$ and no more than $10 \mathrm{GPa}$, respectively. The main factor leading to the discrepancy between our simulations and the experimental results is attributed to the strain rate in comparison with the effects of the defects. Most importantly, the double yielding phenomenon occurs for shock wave propagating in $\mathrm{MgO}$ sample with Schottky defects, i.e., homogeneous nucleation of dislocations accompanied by vacancy emission of dislocations, which has never been reported before. This work sheds light on clarifying the contradictory results of $\mathrm{HEL}$ of $\mathrm{MgO}$ in the literature and understanding the plastic deformation mechanism of MgO. The methodology of this study can be used to study the mechanical response of materials, which have a significant potential for use under extreme environment, such as irradiation condition resulting in high level concentration of vacancies.

\section{Conflicts of interest}

There are no conflicts of interest to declare.

\section{Acknowledgements}

This work was supported by the China Postdoctoral Science Foundation under Grant No. 2016M590586; the National Science Foundation of China under Grant No. 11605231, 11674329, 11504381, 11534012 \& NSAF U1230202; the calculations were partly performed in Center for Computational Science of CASHIPS, the ScGrid of Supercomputing Center and Computer Network Information Center of Chinese Academy of Sciences, and partly using Tianhe-2JK computing time award at the Beijing Computational Science Research Center (CSRC).

\section{References}

1 M. Murakami, Y. Ohishi, N. Hirao and K. Hirose, Earth Planet. Sci. Lett., 2009, 277, 123-129.

2 T. S. Duffy, R. J. Hemley and H. K. Mao, Phys. Rev. Lett., 1995, 74, 1371-1374.

3 Z. Q. Wu, R. M. Wentzcovitch, K. Umemoto, B. Li, K. Hirose and J. Zheng, J. Geophys. Res., 2008, 113, B06204.

4 K. Jin, X. Z. Li, Q. Wu, H. Y. Geng, L. C. Cai, X. M. Zhou and F. Q. Jing, J. Appl. Phys., 2010, 107, 113518.

5 T. J. Ahrens, J. Appl. Phys., 1966, 37, 2532-2541.

6 D. E. Grady, in High Pressure Research: Applications in Geophysics, Academic Press, New York, 1977, p. 389.

7 X. Liu, T. Mashimo, K. Ogata, T. Kinoshita, T. Sekine, X. M. Zhou and W. J. Nellis, J. Appl. Phys., 2013, 114, 243511.

8 G. D. Stevens, L. R. Veeser, P. A. Rigg and R. S. Hixson, AIP Conf. Proc., 2006, 845, 1353-1356.

9 R. S. McWilliams, D. K. Spaulding, J. H. Eggert, P. M. Celliers, D. G. Hicks, R. F. Smith, G. W. Collins and R. Jeanloz, Science, 2012, 338, 1330-1333.
10 D. Alfè, Phys. Rev. Lett., 2005, 94, 235701.

11 A. Aguado and P. A. Madden, Phys. Rev. Lett., 2005, 94, 068501.

12 Z. J. Liu, X. W. Sun, Q. F. Chen, L. C. Cai, X. M. Tan and X. D. Yang, Phys. Lett. A, 2006, 353, 221-225.

13 M. Matsui, J. Chem. Phys., 1998, 108, 3304-3309.

14 D. Cebulla and R. Redmer, Phys. Rev. B: Condens. Matter Mater. Phys., 2014, 89, 134107.

15 S. N. Luo, T. C. Germann and D. L. Tonks, J. Appl. Phys., 2010, 107, 056102.

16 Y. Tang, E. M. Bringa, B. A. Remington and M. A. Meyers, Acta Mater., 2011, 59, 1354-1372.

17 Y. Tang, E. M. Bringa and M. A. Meyers, Acta Mater., 2012, 60, 4856-4865.

18 E. Q. Lin, H. J. Shi and L. S. Niu, Modell. Simul. Mater. Sci. Eng., 2014, 22, 035012.

19 B. P. Uberuaga, R. Smith, A. R. Cleave, F. Montalenti, G. Henkelman, R. W. Grimes, A. F. Voter and K. E. Sickafus, Phys. Rev. Lett., 2004, 92, 115505.

20 T. Hatano, Phys. Rev. Lett., 2004, 92, 015503.

21 J. Marian, J. Knap and M. Ortiz, Acta Mater., 2005, 53, 28932900.

22 R. E. Rudd, Philos. Mag., 2009, 89, 3133-3161.

23 E. M. Bringa, S. Traiviratana and M. A. Meyers, Acta Mater., 2010, 58, 4458-4477.

24 C. J. Ruestes, E. M. Bringa, A. Stukowski, J. F. R. Nieva, G. Bertolino, Y. Tang and M. A. Meyers, Scr. Mater., 2013, 68, 817-820.

25 P. Erhart, E. M. Bringa, M. Kumar and K. Albe, Phys. Rev. B: Condens. Matter Mater. Phys., 2005, 72, 052104.

26 S. Plimpton, J. Comput. Phys., 1995, 117, 1-19.

27 C. L. Kelchner, S. J. Plimpton and J. C. Hamilton, Phys. Rev. B: Condens. Matter Mater. Phys., 1998, 58, 11085.

28 A. Stukowski, V. V. Bulatov and A. Arsenlis, Modell. Simul. Mater. Sci. Eng., 2012, 20, 085007.

29 A. Stukowski, Modell. Simul. Mater. Sci. Eng., 2010, 18, 015012.

30 G. V. Lewis and C. R. A. Catlow, J. Phys. C: Solid State Phys., 1985, 18, 1149-1161.

31 H. K. Mao and P. M. Bell, J. Geophys. Res., 1979, 84, 4533-4536. 32 K. Hirose, N. Sata, T. Komabayashi and Y. Ohishi, Phys. Earth Planet. Inter., 2008, 167, 149-154.

33 S. P. Marsh, LASL Shock Hugoniot Data, University of California Press, Berkeley, CA, 1980, p. 312.

34 S. V. Sinogeikin and J. D. Bass, Phys. Earth Planet. Inter., 2000, 120, 43-62.

35 S. Sarva and S. Nemat-Nasser, Mater. Sci. Eng., A, 2001, 317, 140-144.

36 T. Hatano, Phys. Rev. Lett., 2004, 93, 085501.

37 C. E. Botez, W. C. Elliott, P. F. Miceli and P. W. Stephens, Phys. Rev. B: Condens. Matter Mater. Phys., 2002, 66, 075418.

38 M. Nastasi, J. W. Mayer and J. K. Hirvonen, Ion-Solid Interactions: Fundamentals and Applications, Cambridge University, Cambridge, 2004.

39 S. Minshall, in Response of metals to high velocity deformation, ed. P. G. Shewmon and V. R. Zackay, Interscience, New York, 1961, pp. 249-274.

40 O. E. Jones and J. D. Mote, J. Appl. Phys., 1969, 40, 4920-4928. 